\title{
Note de recherche \\ Nutrition et alimentation des poissons \\ Nutrition et alimentation des poissons : éléments pour un nouveau développement
}

\section{Sadasivam Kaushik}

Inra

UMR 1067 Nutrition, aquaculture

et génomique

Pôle hydrobiologie

64310 Saint-Pée-sur-Nivelle

France

<kaushik@st-pee.inra.fr>

\begin{abstract}
Résumé
Sur le plan mondial, la contribution de l'aquaculture à l'apport protéique alimentaire de l'homme est indéniable. Un des enjeux majeurs est de réduire sa dépendance sur les ingrédients issus de la pêche minotière. De grands progrès sont déjà réalisés dans ce sens.
\end{abstract}

Mots clés : alimentation humaine ; aquaculture ; lipide ; pêches ; protéine animale.

Thèmes : alimentation, consommation, nutrition ; pêche et aquaculture ; productions animales ; recherche scientifique et agronomique.

\section{Abstract \\ Nutrition and feeding of fish: Upcoming developments}

Aquaculture's contribution to the human food basket is undeniable. One major issue however is to reduce its reliance on ingredients derived from feed grade fisheries. Recent research has contributed significantly towards this goal.

Key words: animal proteins; aquaculture; fisheries; human feeding; lipids.

Subjects: animal productions; fishing and aquaculture; food, consumption, nutrition; scientific and agricultural research.

U n des enjeux majeurs de l'aquaculture est d'assurer l'amélioration de l'apport protéique de l'homme, surtout dans un contexte où de très grandes disparités persistent et s'accentuent entre populations humaines. Les contributions relatives de l'aquaculture et de la pêche dans l'approvisionnement de poissons pour la consommation humaine font souvent l'objet de controverses (Pauly et al., 1998 ; Naylor et al., 2000 ; Tidwell et Allan, 2001 ; Alder et al., 2008). Il est cependant clair que sur le plan mondial, l'aquaculture contribue de façon positive à l'apport alimentaire dans la mesure où la grande majorité des poissons d'élevage provient de l'élevage en étangs à faible niveau d'intrants, bénéficiant de la productivité naturelle (FAO, 2007).

Quelles que soient les méthodes et pratiques d'élevage et les espèces concernées, l'apport nutritionnel nécessaire pour une production piscicole donnée (fertilisation adéquate pour améliorer la productivité naturelle ou aliments composés plus ou moins élaborés) constitue la plus grande part des coûts, allant au-delà de $60 \%$ du total des coûts de l'exploitation. La croissance soutenue que connait l'aquaculture aujourd'hui (figure 1) est accompagnée d'une nette tendance vers des systèmes de production semi-intensifs nécessitant une meilleure maitrise des intrants, dont notamment les apports d'aliments. À l'heure actuelle, bien que les aliments composés pour aquaculture ne représentent qu'une faible proportion $(<5 \%) \mathrm{du}$ marché mondial des aliments pour animaux, leur utilisation est en augmentation. Même dans les régions où l'élevage de poissons en étangs avec de faibles niveaux d'intrants constitue la pratique traditionnelle, on assiste à une augmentation de l'utilisation d'aliments composés pour améliorer la productivité piscicole. Le développement d'aliments équilibrés sur les plans nutritionnel et de la durabilité comme sur les plans économique et environnemental est un enjeu majeur pour l'aquaculture, comme pour d'autres 


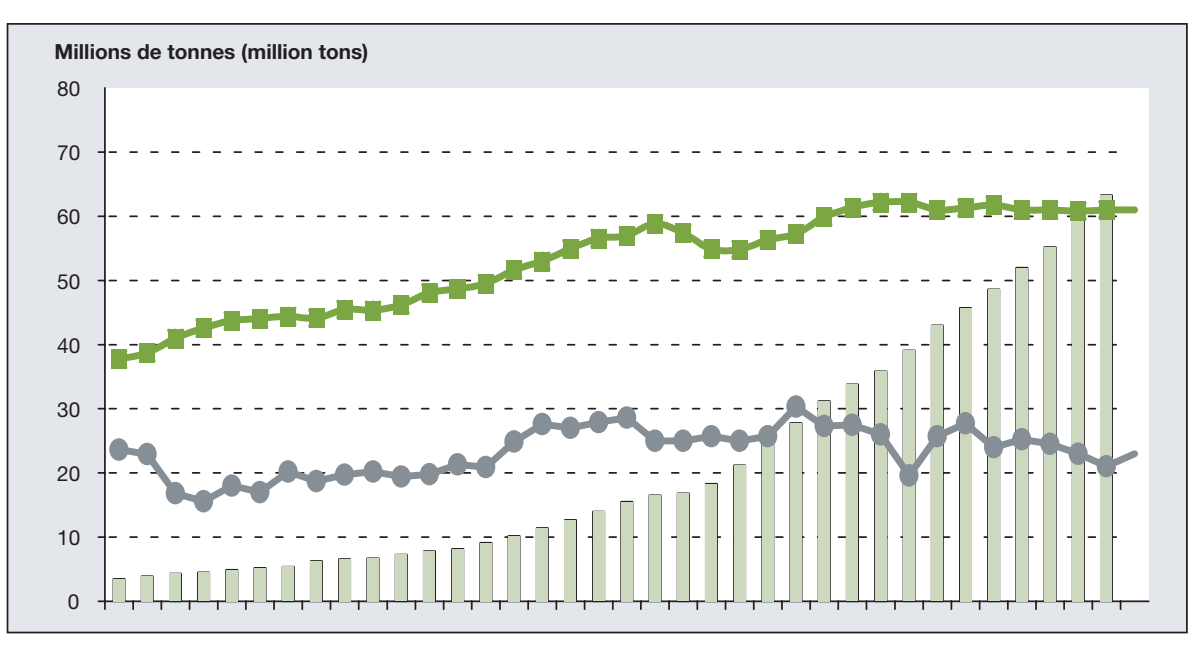

Figure 1. Évolution de la production aquacole (barres verticales) comparée à celle de la pêche pour la consommation humaine directe ( $(\mathbf{)}$ ou la pêche minotière dédiée à la transformation en farines et huiles $(\bullet)$.

Figure 1. Evolution of aquaculture production (vertical bars) compared to wild fishery resources available as food for man ( $\bullet$ ) or used by feed industry for conversion into fishmeal and fish oil (•).

Données en millions de tonnes par an. productions animales. Le défi est de disposer d'aliments permettant de couvrir les besoins nutritionnels, d'assurer le bienêtre physiologique tout au long du cycle de vie (des larves aux géniteurs) des animaux, de maîtriser la valeur nutritionnelle et organoleptique des produits, tout en prenant en compte des enjeux environnementaux tant au niveau des systèmes d'élevage que sur le plan plus global de la gestion de ressources alimentaires. Lorsqu'il s'agit de l'alimentation des poissons et des crustacés d'élevage, un enjeu de taille est de répondre à la question récurrente sur sa dépendance à l'égard des ingrédients d'origine marine: est-il raisonnable de produire du poisson avec du poisson (figure 1)?

Les aliments aquacoles diffèrent des aliments pour animaux terrestres par leur teneur généralement élevée en matières azotées totales et en énergie, ce qui implique en conséquence l'emploi d'ingrédients riches en protéines et en énergie. La couverture des besoins en acides aminés indispensables et en acides gras essentiels des poissons et des crustacés nécessite souvent l'emploi de matières premières issues de la pêche minotière.

Sur les quelques 5,5 millions de tonnes de farines de poissons, l'aquaculture en utilise actuellement (FAO, 2007) plus de la moitié (2,94 millions de tonnes). La répartition de cette utilisation selon le groupe d'espèces montre une forte consommation de farines de poissons pour l'élevage des crustacés et des poissons marins (> $43 \%$ ), des salmonidés $(27 \%)$ et le reste pour l'élevage d'autres espèces comme les cyprinidés, les cichlidés ou les siluriformes (figure 2).
Lorsqu'il s'agit de l'emploi d'huiles de poissons, sur les quelques 900000 tonnes disponibles, l'aquaculture en utilise plus de $90 \%$ avec une répartition, cependant, très contrastée (figure 3) : l'élevage des salmonidés (saumons et truites) consomme plus de $65 \%$ des huiles disponibles, et celui des poissons et des crustacés marins vient ensuite (21\%).

Selon le comité des pêches et de l'aquaculture de la FAO (2008), le plus grand défi pour le développement de l'aquaculture est, en effet, la disponibilité en aliments de bonne qualité en quantités suffisantes ayant peu recours aux ingrédients d'origine marine. Pour répondre à cet enjeu du développement mondial de l'aquaculture tout en limitant l'emploi de matières premières issues de la pêche minotière, les recherches avaient été initiées par nous mêmes (Kaushik et al., 1995, 2004 ; Regost et al., 2003) et par d'autres (Bell et Waagbo, 2008) depuis un certain nombre d'années ; ces recherches ont eu pour objectifs l'optimisation de l'apport azoté, la réduction de l'apport en farines de poissons, la substitution de l'huile de poissons. Les résultats montrent que même pour les espèces qui se situent à un niveau trophique élevé comme les salmonidés ou les poissons

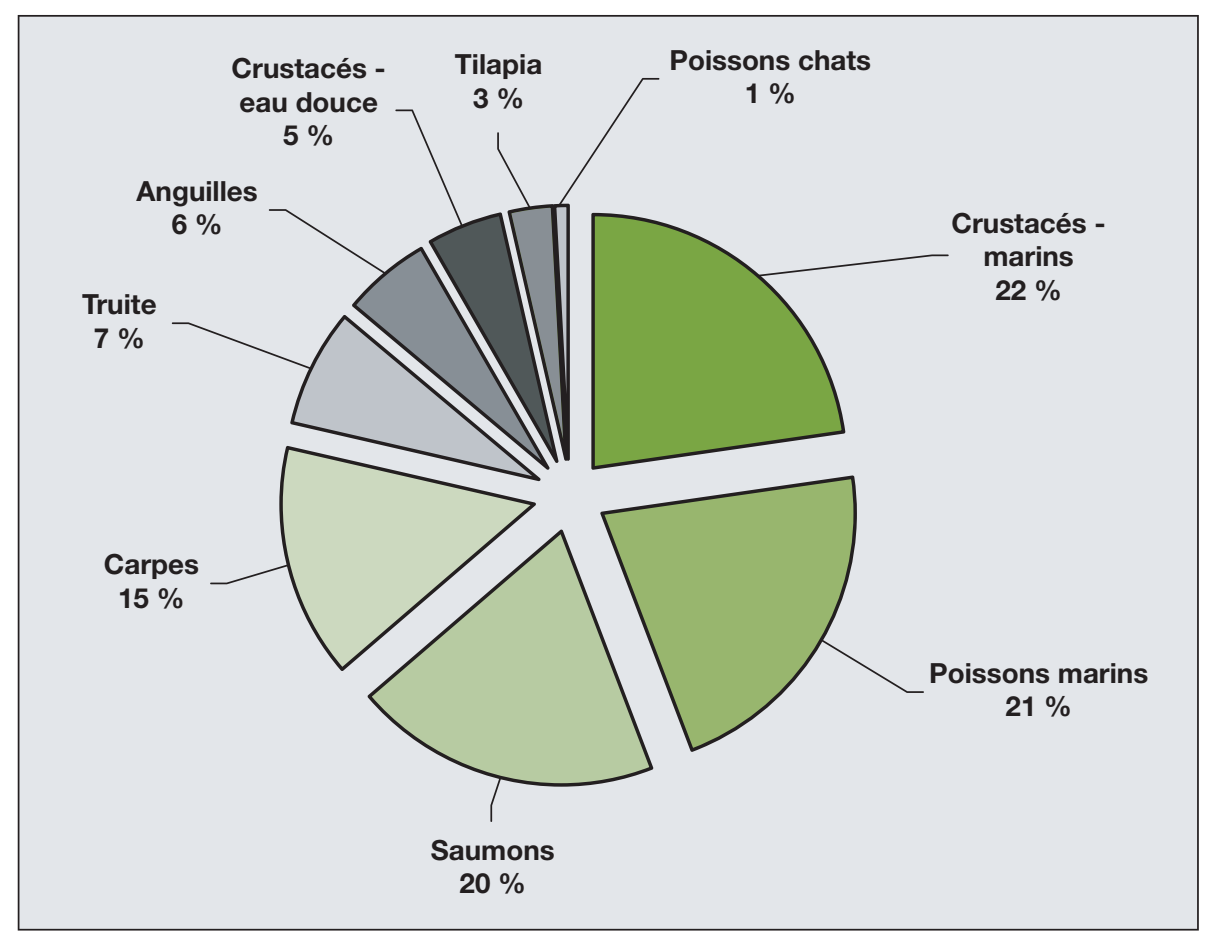

Figure 2. Répartition de l'emploi de la farine de poissons (environ 5,5 millions de tonnes) dans les aliments pour différentes espèces aquacoles (données pour 2006).

Figure 2. Share of available fishmeal (about 5.5 million tons) utilised in feeds for different groups of farmed fish (data for 2006). 


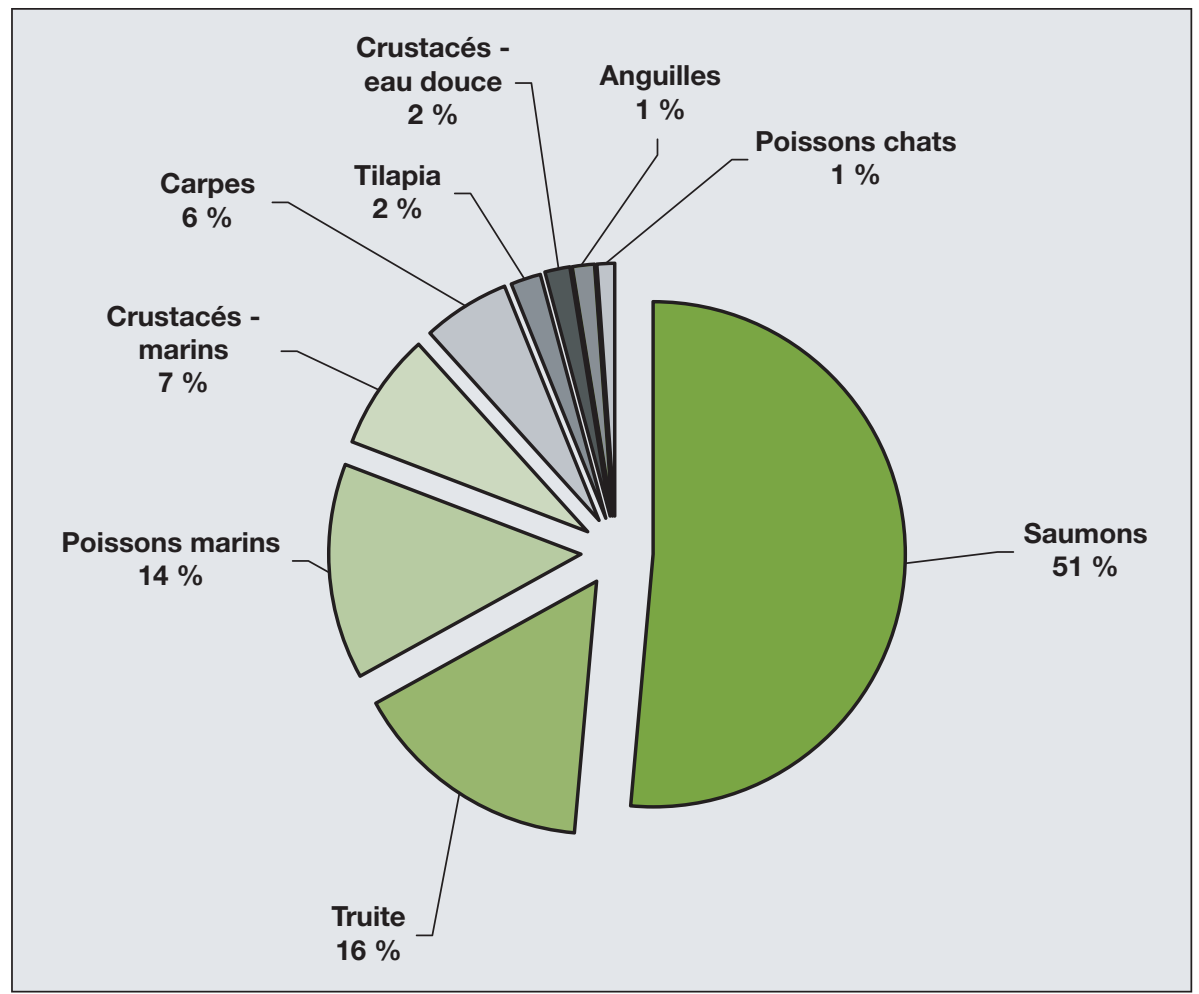

Figure 3. Répartition de I'emploi de I'huile de poissons $(<900000$ tonnes) dans les aliments pour différentes espèces aquacoles (données pour 2006).

Figure 3. Share of available fish oil (900 000 metric tons) utilised in feeds for different groups of farmed fish (data for 2006).

marins, il est possible d'utiliser les aliments ayant des apports réduits en farines et huiles de poissons, sans effets adverses tant sur le plan zootechnique que sur le plan du bien-être physiologique et aussi sur le plan des qualités organoleptiques (Kaushik et al., 1995 ; Bell et Waagbo, 2008). L'évolution des connaissances dans ces domaines et leurs applications dans les pratiques d'élevage, notamment en ce qui concerne

\section{Références}

Alder J, Campbell B, Karpouzi V, Kaschner K, Pauly D. Forage fish: from ecosystems to markets. Annual Review of Environment and Resources 2008 ; 33 : 153-66.

Bell JG, Waagbo R. Safe and nutritious aquaculture produce: benefits and risks of alternative sustainable aquafeeds. In: Holmer $\mathrm{M}$,

Black K, Duarte C, Marba N, Karakassis I, eds. Aquaculture in the Ecosystem. Dordrecht (The Netherlands): Kluwer Academic Publishers Group, 2008.

Corraze G. Alimentation lipidique et remplacement des huiles de poisson par des huiles végétales en pisciculture. Cah Agric 2009 ; 18 : 112-8. Doi : 10.1684/agr.2009.0285

FAO. The state of world fisheries and aquaculture 2006. Rome: FAO, 2007 (162 p).

FAO. Opportunities for addressing the challenges in meeting the rising global demand for food fish from aquaculture. FAO/Committee on fisheries. Meeting of the sub committee on aquaculture, Puerto Varas, Chile, oct 2008, (COFI/AQ/IV/2008/6), 2008.

ftp://ftp.fao.org/docrep/fao/meeting/014/ k3060e.pdf.

Kaushik SJ, Cravedi JP, Lalles JP, Sumpter J Fauconneau B, Laroche M. Partial or total replacement of fish meal by soybean protein on growth, protein utilization, potential estrogenic or antigenic effects, cholesterolemia and flesh quality in rainbow trout, Oncorhynchus mykiss. Aquaculture 1995 ; 133 : 257-74.

Kaushik SJ, Coves D, Dutto G, Blanc D. Almost total replacement of fish meal by plant protein sources in the diet of a marine teleost, the European seabass, Dicentrarchus labrax. Aquaculture $2004 ; 230$ : 391-404.

Médale F, Kaushik S. Les sources protéiques dans les aliments pour les poissons d'élevage. Cah Agric 2009; 18: 103-11. Doi : 10.1684/ agr.2009.0279

Naylor RL, Goldburg RJ, Primavera JH, et al. Effect of aquaculture on world fish supplies. Nature 2000 ; 405 : 1017-24.

Pauly D, Christensen V, Dalsgaard J, Froese R, Torres F. Fishing down marine food webs. Science $1998 ; 279$ : 860-3.

Regost C, Arzel J, Robin J, Rosenlund G, Kaushik SJ. Total replacement of fish oil by soybean or linseed oil with a return to fish oil in turbot (Psetta maxima). Growth performance, flesh fatty acid profile, and lipid metabolism. Aquaculture 2003 ; 217 : 465-82.

Tidwell JH, Allan GL. Fish as food: aquaculture's contribution. EMBO Rep 2001 ; 21 : 958-63. 